\title{
Cushing's Syndrome secondary to ACTH-producing prostate adenocarcinoma: A case report
}

\section{Dr M Scopazzini, Dr J Pomroy \& Dr E Hatfield}

Division of Diabetes, Endocrinology \& Metabolic Medicine, Charing Cross Hospital

Imperial College Healthcare NHS Trust, UK

email:

\section{Introduction}

Cushing's Syndrome refers to the clinical manifestations that result from excess levels of circulating steroid ${ }^{1}$.

Ectopic secretion of ACTH accounts for about $10 \%$ of cases of endogenous hypercortisolaemia ${ }^{1-2}$. Lung tumours account for the vast majority of these ${ }^{2}$.

There are a small number of cases described of ACTH secretion from prostate cancer ${ }^{2-4}$, but we have not found any other cases of histologically confirmed prostatic adenocarcinoma with neuroendrocrine differentiation, which secreted clinically significant levels of ACTH. We present such a case in a 62-yearold man.

\section{Case Report}

- 62 year-old male presenting with a two-week history of intermittent confusion, plethora and left arm weakness on a background of rapidly progressing metastatic prostate adenocarcinoma diagnosed eight months earlier. Poor response to conventional prostate cancer chemotherapy (Bicalutamide $\rightarrow$ anti-androgen $(\mathrm{LHRH}) \rightarrow$ Docetaxel);

- Histology report (Aug 2015) described a Gleason 5+4 adenocarcinoma with neuroendocrine differentiation;

- Referred to the admitting medical team with a history of fast AF, new hyperglycaemia and hypokalaemia refractory to intravenous potassium replacement - initial K 2.1;

- Managed as Cushing's Syndrome based on excessive random cortisol and ACTH, which did not suppress following overnight dexamethasone suppression test;

- Metyrapone + Ketoconazole initiated within 48 hours of presentation.

\section{Investigation and management}

Initial investigations: ECG - prolonged QT (550ms); BM - 17.1 (previously non-diabetic); MRI spine - malignant root compression of C6 nerve root. Initial management: Aggressive potassium and magnesium replacement; Metyrapone and Ketoconazole started to treat

hypercortisolaemia; anti-diabetic medications initiated; urgent oncology review and discussion at neuroendocrine MDT for further management.

\section{Cortisol, ACTH and K levels}

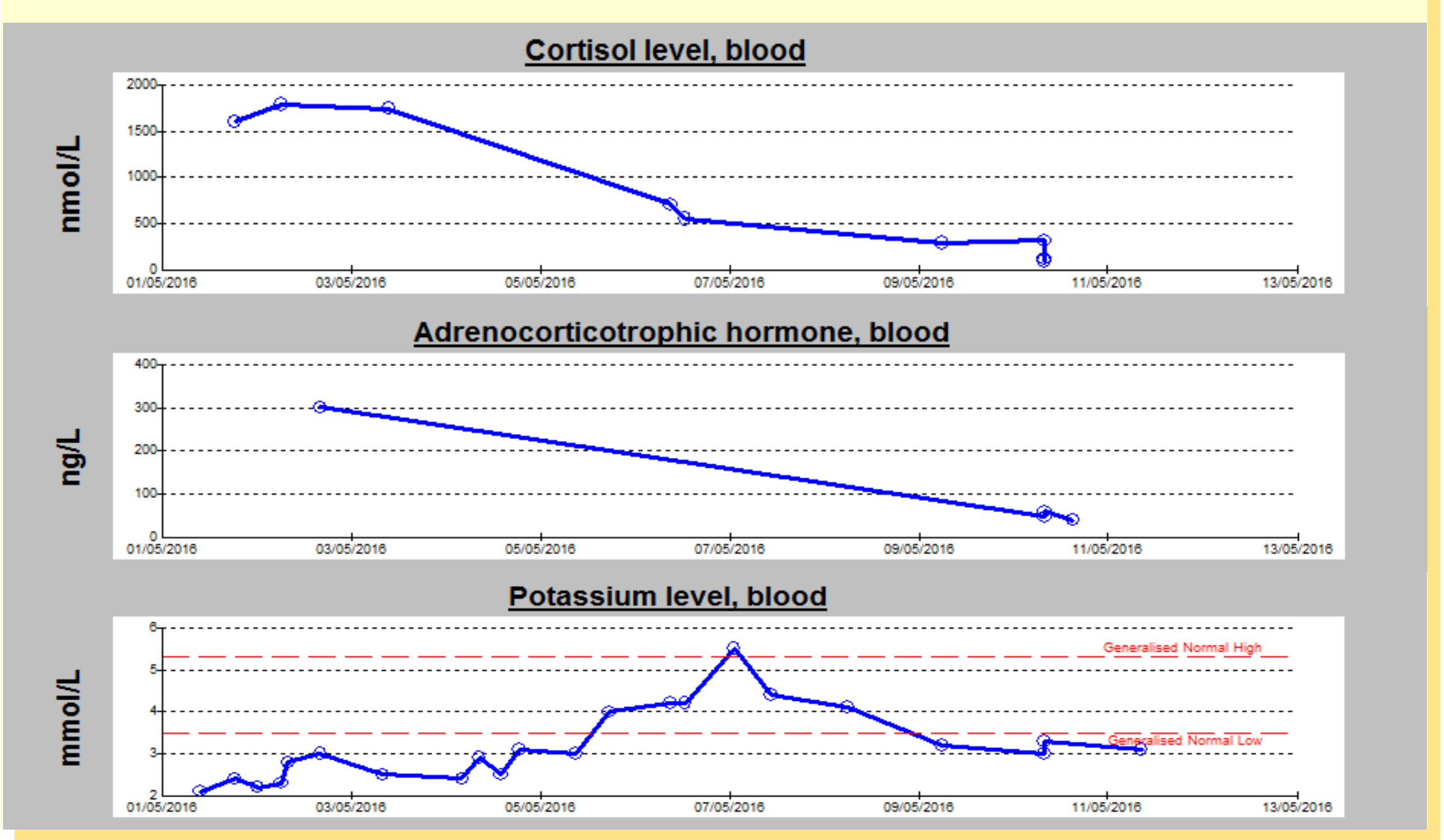

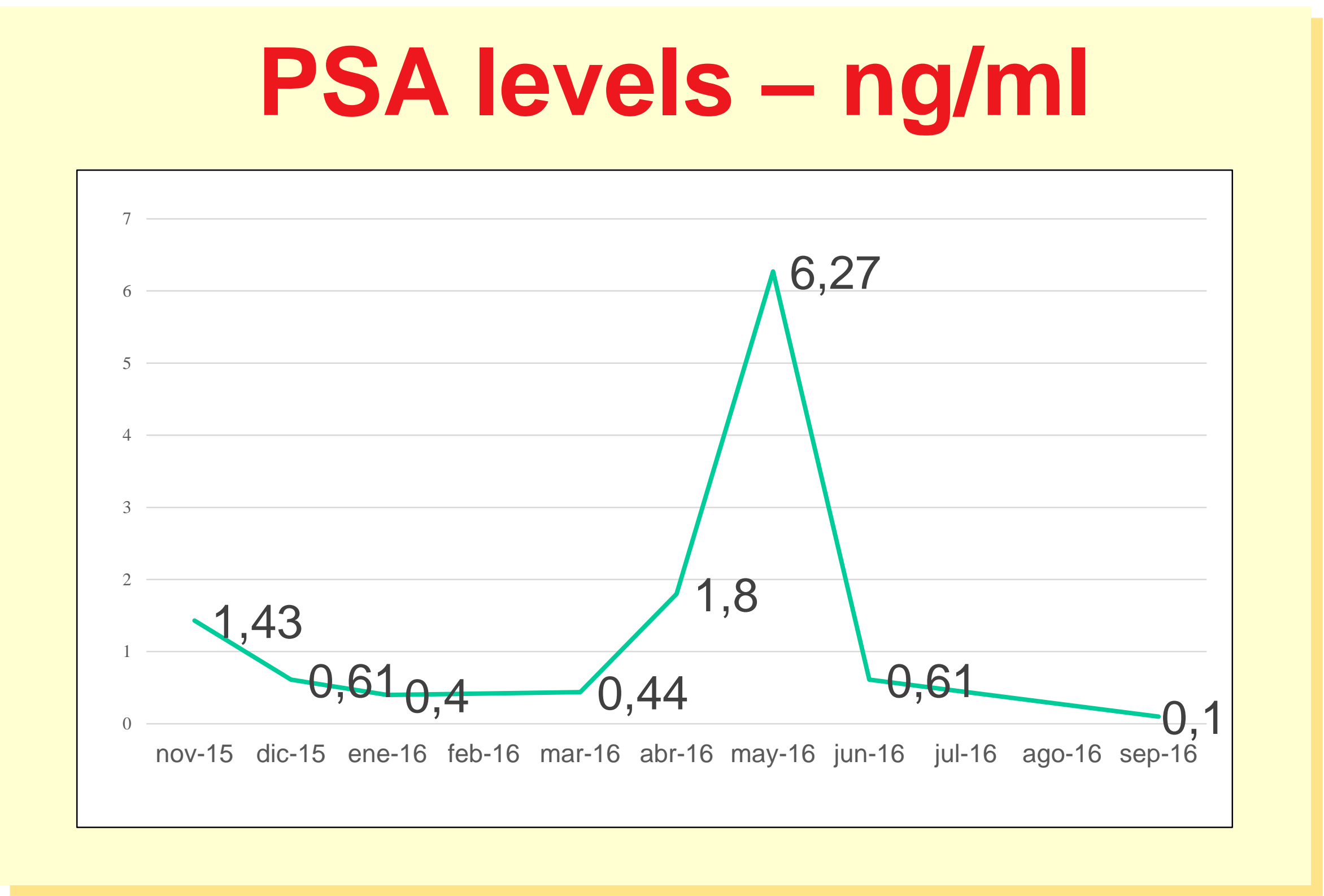

Histology
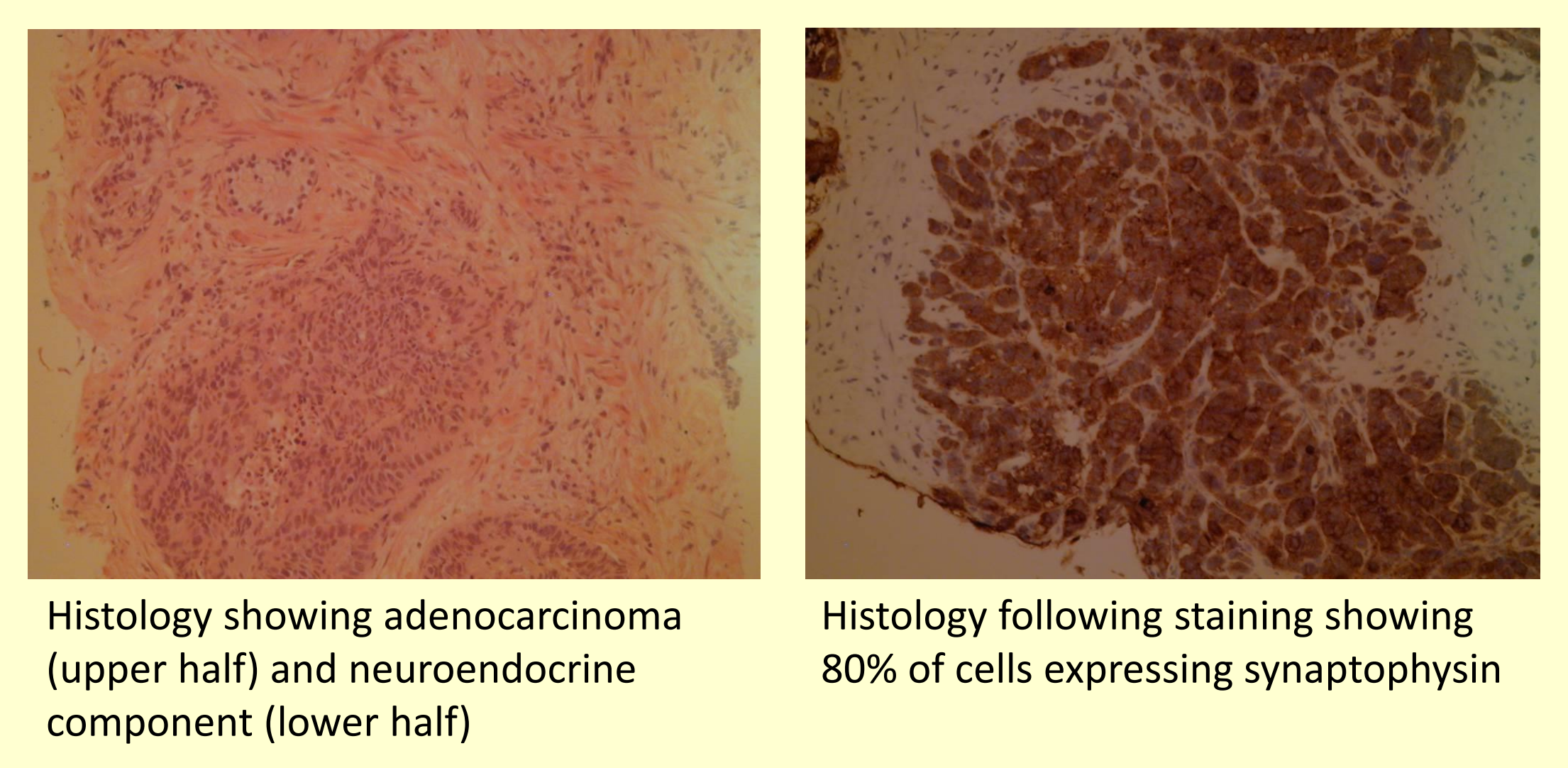

\section{Outcome}

Once we introduced Metyrapone and Ketoconazole, our patient's cortisol was successfully suppressed - his potassium normalised, and his hyperglycaemia was brought under control.

We reviewed his histology, which was negative for retrospective ACTH staining. He was not biopsied again. We discussed his management extensively at neuroendocrine, oncology and endocrine surgery MDTs: he was not felt to be a candidate for surgical intervention. His chemotherapy was subsequently changed from conventional prostate cancer chemotherapy to Etoposide-Carboplatin; now in his sixth cycle, he continues to show a sustained partial response - his ACTH and PSA are suppressed and his spinal metastases have regressed.

The prolonged elevation of $\mathrm{ACTH}$ and associated high cortisols suppressed his HPA axis, and he now requires replacement hydrocortisone. 